die praktische Politik hineingewirkt. Auffällig ist jedoch die immer wiederkehrende Feststellung, dass von einer richtigen Schulbildung nicht die Rede sein könne. Die als „Freiburger" „Heidelberger“ und „Marburger Schule“ bezeichneten wissenschaftlichen Denkzweige sind bekannt, doch eine „Berliner“ Schule hat sich nicht herausbilden können, „schon wegen des Fehlens eines Gravitationszentrums“ (S. 13).

Den Herausgebern ist die Komposition eines durchaus als monumental zu bezeichnenden Bandes gelungen, der sich gewiss als Standardwerk behaupten wird. Der Gesamtertrag der Porträts ist hoch. In der Verdichtung von Namen, politischen Entwicklungen der Bundesrepublik, Entstehungsspuren des Faches sowie von Forschungsgegenständen wirken die Kapitel mitunter wie ein Pumpwerk der Politikwissenschaft. Hier liegt ein biografisches Handwörterbuch vor, das auch Auskunft über die Entwicklung des Faches selbst gibt. Dieses Buch ist wie Goldstaub für jede Bibliothek eines politikwissenschaftlichen Seminars.

Helge F. Jani

Politische Biographien: empfehlenswerte Sammlung für die politische Bildung

Rahmig, Jürgen: Politiker mit Leib und Seele, Oertel + Spörer Verlag, Reutlingen 2013, 292 Seiten mit Abbildungen, € 19,90.

Die Absicht des Autors ist lobenswert: Ausgehend von dem verbreiteten Vorurteil, dass sich immer weniger junge Menschen für Politik interessieren, hat sich Jürgen Rahmig vorgenommen, „Lust auf Politik“ (Rückseitentext) zu machen. Die Prämisse, von der er ausgeht, ist auch richtig, wie einschlägige Untersuchungen zur Politikverdrossenheit zeigen: Jugendliche sind nicht generell an Politik desinteressiert - nur für Parteipolitik können sie sich nicht erwärmen. Sie wollen sich politisch beteiligen, aber vorzugsweise an den so genannten „unkonventionellen“ Formen der Politik. Die Idee des Politikredakteurs beim Reutlinger General-Anzeiger hat Charme: Indem er 19 prominente Politiker Baden-Württembergs in Kurzporträts vorstellt, sie über ihren Weg in die Politik, ihre (politischen) Leidenschaften und Abneigungen erzählen lässt, möchte er junge Menschen auch für die „konventionelle“ Parteipolitik begeistern, die derzeit nicht in besonders hohem Kurs steht. Sie sollen erkennen, dass auch Politiker „Menschen sind wie du und ich, mit ihren Schwächen und Stärken, ihren Vorlieben und Antipathien“ (Klappentext).

Nicht zufällig macht mit der grünen Bundestagsabgeordneten Agnieszka Brugger die jüngste der vorgestellten Politikerinnen den Anfang: Sie zog bereits 2009 mit 24 Jahren erstmals in den Bundestag ein, damals noch unter ihrem Mädchennamen Malczak. Agnieszka Brugger hat Politikwissenschaft in Heidelberg studiert, sie trägt Piercings an Nase und Unterlippe, ist insofern durchaus eine unkonventionelle Abgeordnete. Von Menschen im Wahlkampf darauf angesprochen, entgegnete sie: „(B)ei einer Politikerin ist wichtig, was aus dem Mund rauskommt und nicht, was am Mund dran ist“ (S. 14). Die Bundestagsabgeordnete entspricht auch sonst nicht typischen Klischees: Sie ist gebürtige Polin (ihre Eltern waren Spätaussiedler), Vegetarierin - was für Grüne so ungewöhnlich aber auch nicht ist - und war in der Jugend als einzige Frau Mitglied in einem Boxverein. Bemerkenswert 
ist auch, dass es Brugger gleich in ihrem ersten Jahr gelang, Mitglied im Verteidigungsausschuss und abrüstungspolitische Sprecherin ihrer Fraktion zu werden.

In ihrem politischen Idealismus und friedenspolitischen Engagement trennt die junge Abgeordnete nicht viel von dem ältesten hier vorgestellten Politiker, dem 1926 in Ulm geborenen Erhard Eppler. Eppler wurde noch mit 16 Jahren in den Zweiten Weltkrieg eingezogen und lernte dort, „was Hunger bedeutet“ (S. 57). In die Politik geriet der angehende Gymnasiallehrer durch den Widerstand gegen Konrad Adenauers Politik der Westintegration. Weil die damalige Bundesregierung die deutschlandpolitischen Stalin-Noten vom März 1952 nicht auslotete, trat Eppler der Gesamtdeutschen Volkspartei Gustav Heinemanns bei. 1956 wechselte er auf Anraten Fritz Erlers zur SPD und machte dort Karriere: 1961 bis 1976 Mitglied des Deutschen Bundestages, 1968 bis 1974 Bundesminister für wirtschaftliche Zusammenarbeit, 1973 bis 1981 Landesvorsitzender in Baden-Württemberg, 1975 bis 1991 Leiter der SPD-Grundwertekommission. Auch als evangelischer Kirchentagspräsident (1981 bis 1983 und 1989 bis 1991) machte Eppler von sich reden. Als Finanzpolitiker dagegen ist Eppler, Ikone der SPD-Linken, der Öffentlichkeit nicht in Erinnerung geblieben. Dabei hat er auf diesem Politikfeld nach eigenem Bekunden einen seiner größten politischen Erfolge erzielt: Der bis heute geltende reduzierte Mehrwertsteuersatz auf Verlagserzeugnisse wurde 1966 von Eppler und dem Finanzausschussvorsitzenden Otto Schmidt (CDU) durchgesetzt (vgl. S. 62 f.).

Mit Brugger und Eppler wurden hier die jüngste und der älteste Politiker aus Rahmigs Buch knapp vorgestellt. Diese Auswahl erfolgte willkürlich - nicht anders als die Auswahl der Politiker durch den Autor. Dennoch hat Rahmig durchaus darauf geachtet, einen für „das Ländle“ annähernd repräsentativen Querschnitt an Politikern auszusuchen: Von den 19 Politikern gehören acht der CDU an (Elisabeth Jeggle, Oswald Metzger, Günther Oettinger, Hermann Schaufler, Lothar Späth, Gerhard Stratthaus, Erwin Teufel, Annette Widmann-Mauz), sechs der SPD (Herta Däubler-Gmelin, Erhard Eppler, Nicolette Kressl, Nils Schmid, Dieter Spöri, Beate Weber-Schuerholz), drei der FDP (Walter Döring, Helmut Haussmann, Klaus Kinkel) und zwei den Grünen (Agnieszka Brugger, Rezzo Schlauch). Dies entspricht in etwa - bezogen auf die Nachkriegszeit - dem politischen Gewicht der Parteien in Baden-Württemberg. Auch der Frauenanteil ist sowohl für das Land (32 Prozent) als auch für die jeweiligen Parteien (SPD und Grüne jeweils 50 Prozent, CDU 25 Prozent, FDP null Prozent) annähernd repräsentativ. Selbst die Altersverteilung der 19 ausgewählten Politiker scheint insofern die tatsächlichen Verhältnisse angemessen abzubilden, als die Kohorte der über 65-Jährigen mit zwölf Politikern (63 Prozent) deutlich überrepräsentiert ist.

Dem vielfach diagnostizierten Problem der Überalterung der Parteien kann also auch Rahmigs Buch schon von seinem Design her nicht völlig entfliehen. Er muss seine Zielgruppe „der Jungen“ ansprechen, indem er ihnen das Leben „der Alten“ interessant macht. Darin kann, muss aber kein Widerspruch liegen. Die gewählte Form des biographischen Interviews lässt auch die älteren Politiker durchaus jung erscheinen, sofern sie im Alter offen und neugierig geblieben sind. Das lässt sich von erstaunlich vielen der hier Porträtierten sagen: von Lothar Späth beispielsweise, der nach seinem Rücktritt als Ministerpräsident eine neue Karriere in der Wirtschaft - und als Fernsehmoderator - begann; von Nachfolger Erwin Teufel, der sich als Senior den Wunsch erfüllte, Philosophie zu studieren; oder von der früheren Europa-Abgeordneten und Heidelberger Oberbürgermeisterin Beate WeberSchuerholz, die nach überstandener Krebserkrankung im Alter von fast 70 Jahren noch einmal heiratete und jetzt zwischen Deutschland und Kanada hin- und herpendelt. 
Dem Buch und seinem Autor ist zu wünschen, dass es seine Zielgruppe erreicht. Es vermittelt interessante, oftmals auch unbekannte Details aus dem Leben (ehemals) prominenter baden-württembergischer Politiker und vermag in seinen lebendigen, anschaulich erzählten biographischen Porträts zu zeigen, dass Politiker keineswegs aalglatt sein müssen, sondern in der Regel ganz normale und sympathische Menschen sind. Damit ist heutzutage - angesichts der stellenweise grassierenden Politikerverachtung - schon viel gewonnen. Für die politische Bildung junger - und auch älterer - Erwachsener kann der Band also durchaus empfohlen werden. Für wissenschaftliche Zwecke dagegen ist es nur von begrenztem Wert - aber dies, war auch nicht der Anspruch des Autors.

Patrick Horst

\section{Parlamentarische Kontrolle von EU-Angelegenheiten: differenziertes Bild aus Wissen- schaft und Praxis}

Eberbach-Born, Birgit, Sabine Kropp, Andrej Stuchlik und Wolfgang Zeh (Hrsg.): Parlamentarische Kontrolle und Europäische Union (Studien zum Parlamentarismus, Bd. 19), Nomos Verlagsgesellschaft, Baden-Baden 2013, 448 Seiten, € 69,-.

Parlamentarische Kontrolle ist, so Wolfgang Zeh, „ein Teil der Mechanismen, mit denen jene Hemmung und Begrenzung staatlicher Macht gewährleistet werden soll“ (S. 5). Sie zählt für Birgit Eberbach-Born und Sabine Kropp „zu den Kernfunktionen von Parlamenten in modernen Demokratien“" (S. 13). Kontrolle ist umso wichtiger, je mehr die Gesetzgebung an Bedeutung verliert, so Sven T. Siefken (S. 50). So lag es nahe, die Funktion der Kontrolle von Europasachen auf den Ebenen der EU, der Mitgliedstaaten und Regionen zu behandeln. Dazu dienen in vier Abschnitten 17 Aufsätze von 19 Wissenschaftlern und Praktikern: fünf Lehrstuhlinhaber, je sieben wissenschaftliche Mitarbeiter und wissenschaftlich tätige Praktiker.

Ins Thema führen Wolfgang Zeh sowie Birgit Eberbach-Born und Sabine Kropp ein. Im Abschnitt Konzepte und Problemdimensionen präsentieren Werner J. Patzelt Begriffe, Leitgedanken und Erscheinungsformen parlamentarischer Kontrolle, Sven T. Siefken ein Konzept für vergleichende Analyse und Gabriele Abels Gedanken zu parlamentarischer Kontrolle im EU-Mehrebenenparlamentarismus, wobei der Fokus „nicht auf einzelnen Parlamenten, sondern auf der Europäisierung des Parlamentarismus" liegt (S. 81). Im umfangreichen Abschnitt zum Bundestag behandeln Sven Hölscheidt die Kontrolle der EuroKrise, Fabian Schulz und Markus Broich den „Bericht zweier Praktiker aus dem Maschinenraum der Zusammenarbeit von EP und Deutschem Bundestag“ (S. 134), Kristin Rohleder und Ulrich Schöler die Herausforderungen für den Wissenschaftlichen Dienst des Bundestags, Sabine Kropp Information und Kontrolle im Parlament, Danny Schindler und Sven T. Siefken die Wahlkreisarbeit als Teil parlamentarischer Kontrolle sowie Daniel Rölle die Einstellung der Bevölkerung zu Bundestag und Europäischem Parlament.

Der Abschnitt zu den Landtagen bringt Aufsätze von Werner Reutter zur Transformation des „neuen Dualismus“, nämlich parlamentarische Kontrolle, Gewaltenteilung und EU, 